\title{
Experimental Studies on Swirling Flow in a Vertical Circular Tube
}

\author{
Tae-Hyun Chang ${ }^{\dagger} \cdot$ Chang-Hoan Lee ${ }^{1}$ \\ (Received October 17, 2011; Revised November 8, 2011; Accepted November 16, 2011)
}

\begin{abstract}
Swirling flows are related to the spiral motion in the tangential direction in addition to the axial and radial direction using several swirl generators. These type of flows are used in combustion chambers to improve flame stability, heat exchanger to enhance heat transfer coefficients, agricultural spraying machines and some vertical pipes to move slurries or transport of materials. However, only a few studies three dimensional velocity profiles in a vertical pipe have been reported. In this present paper, 3 dimension particle image velocimetry(PIV) technique was employed to measure the velocity profiles in water along a vertical circular pipe with Reynolds number from 6000 to 13,000. A tangential inlet condition was used as the swirl generator to produce the required flow. The velocities were measured with swirling flow in the water along the test section using the PIV technique.
\end{abstract}

Key words : reversal core, swirl intensity, swirl generator

\section{Introduction}

In recent years, considerable attention has been paid to swirling flow through circular or rectangular tubes. During the past three or four decades, the characteristics of swirling flow has been studied extensively because of its great technological and scientific importance. It is well known that swirling flow improves heat transfer in a pipe flow. Thus, this flow has been studied widely in the gasoline, diesel engine, swirl jet, spray or flame, atomizer, in the cylindrical pipe, annuli, as well as in the $90^{\circ}$ degree and $180^{\circ}$ circular bend. using a number of visualization techniques.

Nuttall (1953) conducted an experimental in a vertical circular tube using guide vanes to produce swirling flow at Reynolds number $3 \times 10^{4}[1]$. Results from this study show that the reverse water flow occurs at the center of the pipe with swirl and discharge rate. As the swirl intensity increases, the axial velocity becomes negative at the center of the test tube. However, the axial velocity is positive at the higher swirl intensity near the tube wall

Binnie et al. (1957) conducted experimental studies on the different diameters of the test sections[2].

They attempted to examine the pressure and velocity distributions inside a convergent nozzle that discharges water downwards under pressure. A volute for swirling flow was employed in the test section. Results show two large departures from the inviscid flow. These events were discovered when permanganate solution were injected into the nozzle using a hyperdermic tube inserted through a pressure tapping.

Binnie (1962) used a Perspex tube(internal

\footnotetext{
† Correspording Author(Senior Research Fellow of RESEAT, Korea Institute of Science and Technology Information E-mail:changtae@reseat.ac.kr, Tel: 055-2465-1279)

1 Information Research Team, Principal Researcher, Korea Institute of Science and Technology Information

This paper is extended and updated from the short version that apeared in the Proceedings of the International symposium on Marine Engineering and Technology (ISMT 2011), held at BEXCO, Busan, Korea on October 25-28, 2011.
} 
diameter $=2 \mathrm{in}$ and wall thickness $=0.25 \mathrm{in}$ ) and $\mathrm{a}$ volute to generate the swirling flow inside the test tube[3]. The flow was adjusted by the supply valves. The most interesting vortex collapse was observed when the pressure head was approximately $9 \mathrm{ft}$.

Wood et al. (2001) detailed the improved particle distributions, that is, particle and the predicted reduction in wear rates[4]. Visualization studies using stimulant particle-laden liquids augmented computational modeling of the flow patterns. The authors have shown potential benefits for the strategic swirl generation in commercial slurry pipelines.

Mak et al. (2007) examined effect of swirl on the flow characteristics of sudden axisymmetric expansion chamber with an expansion ratio of 2.5[5]. They employed particle image velocimetry(PIV) to capture the instantaneous flow field using a guider vane at $\operatorname{Re}=10,000$ and swirl number was varied from 0 to 0.65 . The finding shows that increasing the swirl number further to 0.65 results in the reverse of flow at the centerline because of the vortex breakdown.

Shyy et al. (2009) recently studied the two-phase structure, pressure drop and heat transfer for the concurrent air-water slug flows in the vertical tube with swirl[6]. High frame-rate videography was used to visualize the two-phase flow with swirl to measure the traditional velocity of the Taylor bubble. They concluded that as the slug flow traverses through the spirally arranged spikes, the Taylor bubble and liquid are accordingly twisted by the swirl developed in Taylor bubble.

To date, little information this phenomenon can be derived in the related literature. In addition, only a few reports on the investigation of the characteristics of velocity with a swirling flow in the vertical circular pipe using PIV techniques have been published. The present study reports the results of velocities profiles with and without swirl.

\section{Experimental Apparatus}

\subsection{Swirl generator}

The swirling generator was fabricated using an acryl tube (outer diameter $=60 \mathrm{~mm}$ ), in which eight holes (diameter $3.0 \mathrm{~mm}$ ) were drilled at $45^{\circ}$ intervals from the outer to inner tangential direction.

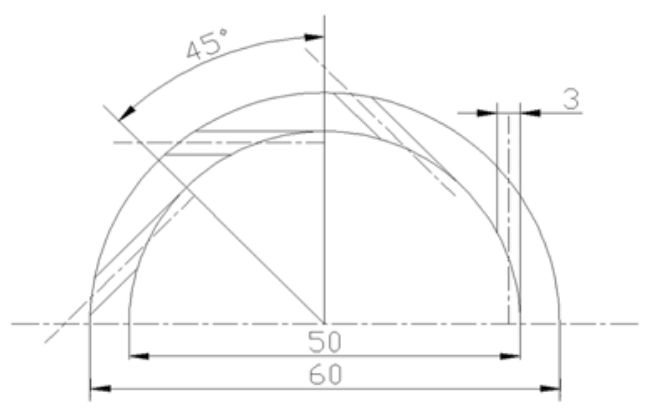

Figure 1: Schematic diagram of the swirl generator

The swirl generator was designed to be able to move in the swirl chamber to adjust the swirl intensity. A detailed diagram of the cross section of the swirl generator is illustrated Figure 1.

\subsection{The test pipe}

The pipe consisted of the swirl generator, test sections, water pump and the water tank (Figure 2).

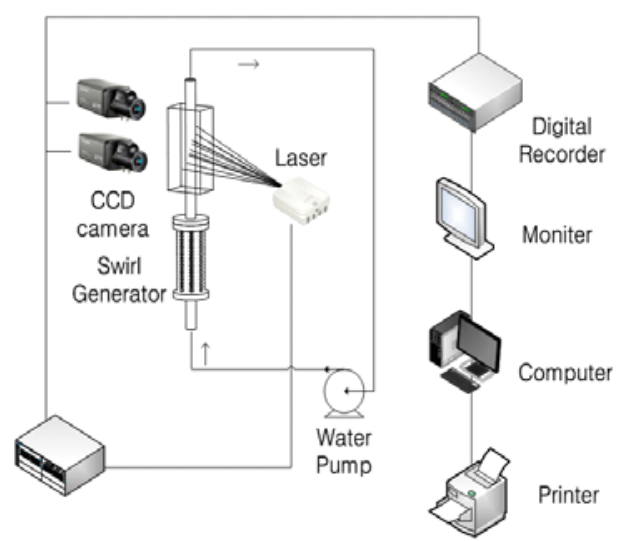

Figure 2: Schematic diagram of the experimental apparatus and PIV system. 
The test pipe consisted of an acryl pipe (internal diameter $=40 \mathrm{~mm}$, length $=1300 \mathrm{~mm}$, and thickness $=4 \mathrm{~mm}$ ). The water tank and the circulating water pump were installed outside the test section.

Water was used as the working fluid, which can be sequentially circulated through the swirl generator, the test pipe and the water tank. A square box was installed, which recovered the refracted light waves from the visualized section of the flow, to prohibit the refractive effects of the circular pipe according to results. For the non-swirling flow experiment, the swirling generator and swirl chamber were dismantled and connected by a guide tube having the same diameter.

\subsection{Stereoscopic PIV system}

A hybrid SPIV has been used for the measurements, in which a geometric transformation has been adopted to consider the refraction and the aberration of the lens and the camera calibration method used for the 3D-PTV measurement

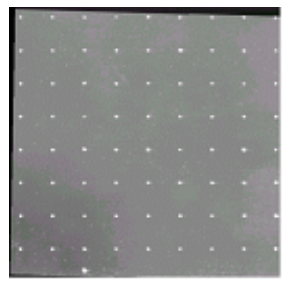

Image of camera 1 Image of camera 2

(a) before transformation
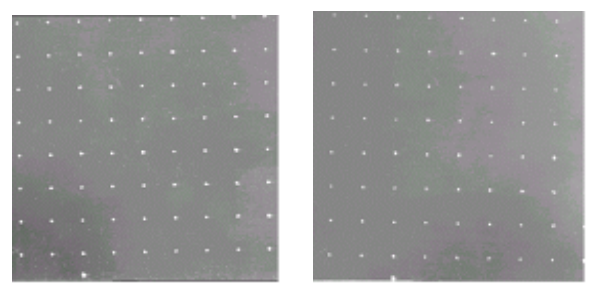

(b) after transformation

Figure 3: Calibrator image before and after transformation developed by Doh et al. (2004)[7]. Soloff et al. (1997) used a mapping function for camera calibration. In that case, the distortion effect of the curved wall of the experimental is considered in the mapping function[8]. On the other hand, the calibration method in this study is based upon the conventional pinhole model, the images viewed through a curved wall pipe are apt to be distorted larger than in the case of mapping function. To reduce the distortion effect, a two-dimensional image transformation on each camera image, warping has been carried out before using the original images for the camera calibration as shown in Eq. (1).

$x=\frac{a_{11} x_{s}+a_{12} y_{s}+a_{13}}{a_{31} x_{s}+a_{32} y_{s}+1} \quad y=\frac{a_{21} x_{s}+a_{22} y_{s}+a_{23}}{a_{31} x_{s}+a_{32} y_{s}+1}$

$x, y:$ coordinates before transformation

$x_{s}, y_{s}$ : coordinates after transformation

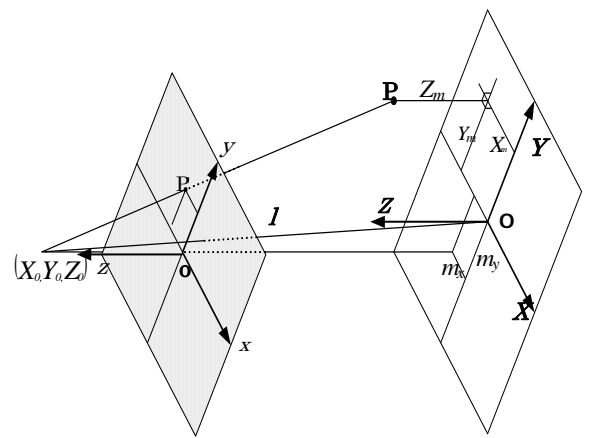

Figure 4: The camera parameters on the absolute coordinate and the photographical coordinate

The calibrator image is shown in Figure 2 before and after this transformation. In this study, the image at the center physical plane $(Z=0 \mathrm{~mm})$ was used to calculate the elements of the $a_{i j}$ matrix in Eq. (1). In the ten-parameter method, 10 parameters (6 exterior parameters: $l, a, b, g, m_{x}, m_{y}$, four interior parameters: $\left.c_{x}, c_{y}, k_{1}, k_{2}\right)$ are obtained. $(a, b$, g) represents the tilting angles of the photographic coordinates axes against the absolute axes. 
Figure 4 shows a coordinate relation when the photographical axes are set parallel to the absolute coordinate by the tilting angles $(a, b, g) .(X, Y, Z)$ represents the absolute coordinate, and $(x, y, z)$ represents the photographical coordinate of the image centroid of the calibration targets. The $l$ means the distance between the origin $O(0,0,0)$ and the principal point $\left(X_{0}, Y_{0}, Z_{0}\right)$ of the camera.

The coordinate $\left(X_{m}, Y_{m}, Z_{m}\right)$ represents the point $\mathrm{P}$ position of the calibrator when the camera coordinate is rotated with the tilting angles to make the collinear set in one line as shown in Figure 4. The $m_{x}$ and $m_{y}$ means the point at which the normal vector from the origin $\mathrm{O}\left(X_{0}, Y_{0}, Z_{0}\right)$ of the camera coordinate meets with the $X-Y$ plane.

The collinear equation for every point between the two coordinates is expressed as Eq. (2). The $c_{x}$ and $c_{y}$ are the focal distances for $x$ and $y$ components of the coordinate. $\Delta x$ and $\Delta y$ are the lens distortions as expressed as Eq. (3). The Eq. (2) can be converted to the following Eq. (4).

$$
x=c_{x} \frac{X_{m}-m_{x}}{\sqrt{l^{2}-m_{x}^{2}-m_{y}^{2}}-Z_{m}}+\Delta x \quad y=c_{y} \frac{Y_{m}-m_{y}}{\sqrt{l^{2}-m_{x}^{2}-m_{y}^{2}}-Z_{m}}+\Delta y
$$

$$
\begin{aligned}
& \Delta x=(x / r) \times\left(k_{1} r^{2}+k_{2} r^{4}\right), \quad \Delta y=(x / r) \times\left(k_{1} r^{2}+k_{2} r^{4}\right), \\
& r=\sqrt{x^{2}+y^{2}}
\end{aligned}
$$

$$
F=C_{x} \frac{X_{m}-m_{x}}{\sqrt{l^{2}-m_{x}^{2}-m_{y}^{2}}-Z_{m}}-(x-\Delta x)=0,
$$$$
G=C_{y} \frac{Y_{m}-m_{y}}{\sqrt{l^{2}-m_{x}^{2}-m_{y}^{2}}-Z_{m}}-(y-\Delta y)=0
$$

Once all camera parameters are obtained, the relations between the photographic coordinate and the absolute coordinate of the target image or the particle image can be expressed as the following Eq. (5).
$\left[\begin{array}{c}X \\ Y \\ Z\end{array}\right]=M_{M}^{-1}\left[\begin{array}{l}X_{m} \\ Y_{m} \\ Z_{m}\end{array}\right]$

Where, $M_{M}$ is the matrix for the rotational transformation is expressed in the form of Eq. (6).

$$
\begin{aligned}
& X_{m}=\frac{x-\Delta x}{c_{x}} t+m_{x}, \quad Y_{m}=\frac{y-\Delta y}{c_{y}} t+m_{y}, \\
& d-Z_{m}=t \quad, \quad d=\sqrt{l^{2}-m_{X}^{2}-m_{y}^{2}}
\end{aligned}
$$

If the camera center is set to a vector $\left(X_{0}, Y_{0}\right.$, $Z_{0}$ ), the collinear equation for one target (or particle) can be expressed as $\mathrm{P}(X, Y, Z)=\left(a_{1} r+\right.$ $\left.X_{0}, a_{2} r+Y_{0}, a_{3} r+Z_{0}\right)$. The cross-sectional points constructed from the following two collinear equations for the two cameras are defined as the three-dimensional positions in the absolute coordinate.

$\mathrm{A}(X, Y, Z)=\mathrm{A}\left(a_{11}^{\prime} t+b_{11}^{\prime}, a_{12}^{\prime} t+b_{12}^{\prime}, a_{13}^{\prime} t+b_{13}^{\prime}\right)$ $\mathrm{B}(X, Y, Z)=\mathrm{B}\left(a^{\prime \prime}{ }_{21} s+b^{\prime \prime}{ }_{21}, a^{\prime \prime}{ }_{22} s+b^{\prime \prime}{ }_{22}, a^{\prime \prime}{ }_{23} s+b^{\prime \prime}{ }_{23}\right)$

Here, $r$ and sare obtained by the least square method (LSM). Since the cross-sectional points do not always intersect on one point, the below Eq. (8) is used for the definition of the last three-dimensional position of the targets (or the particles), which implies the center of the shortest distance between the two collinear equations.

$$
\left[\begin{array}{c}
X_{P} \\
Y_{P} \\
Z_{P}
\end{array}\right]=\frac{1}{2}\left\{\left[\begin{array}{c}
X_{A} \\
Y_{A} \\
Z_{A}
\end{array}\right]+\left[\begin{array}{c}
X_{B} \\
Y_{B} \\
Z_{B}
\end{array}\right]\right\}
$$

Where, $X_{A}, \quad Y_{A}$ and $Z_{A}$ represent the absolute coordinates for thecamera A defined by Eq. (7). $X_{B}$, $Y_{B}$ and $Z_{B}$ represent the absolute coordinates for the camera B. After obtaining the positions of the vector grid points (vector start points) in this manner, the three-dimensional vector terminals were 
obtained by making vector addition with the two sets of the two-dimensional vector terminals that were obtained by the conventional gray level cross-correlation method by Kimura (1986) for the two cameras' image[9].

\subsection{Experimental results and discussions}

\subsubsection{Velocity measurement}

The three velocities $\mathrm{Vy}, \mathrm{Vr}$ and $\mathrm{Vz}$ with and without swirl flow are measured along the test section using PIV technique for $\mathrm{Re}=44000$ to 13200. The size of the employed particle is $100 \mu \mathrm{m}$ to $120 \mu \mathrm{m}$ nylon.

The raw images at $\operatorname{Re}=8800$ and $\operatorname{Re}=13000$ are shown in Figure 5 and 6, respectively. The images were taken at 200 frames/s in the test section using two cameras. A vortex core was shown with the swirl in the test tube at $\mathrm{Re}=8800$ and $\mathrm{Re}=13000$. The vortex core increased by increasing the Reynolds number.

In addition, the particles are indicated at a strong flow angle and then moved up along the tube. From this presentation, when swirling flow has a higher Reynolds number or strong swirl, the vortex core becomes bigger in the test section. These phenomena are very similar to the results from the

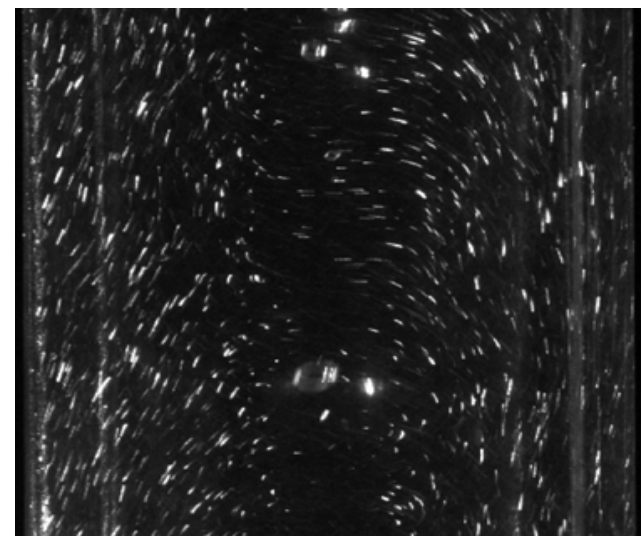

Figure 5: Raw image with swirl for $\mathrm{Re}=8800$ studies of Nuttal [1] and Binnie [2].

Moreover, the size of the vortex core is smaller than that in a horizontal tube.

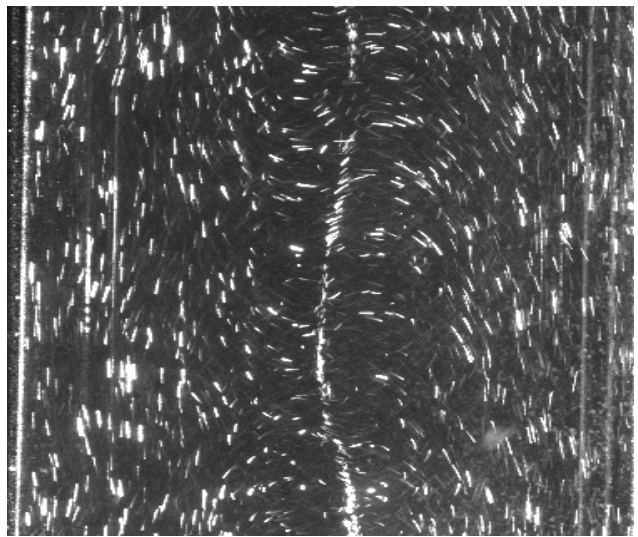

Figure 6: Raw image with swirl for $\mathrm{Re}=13000$

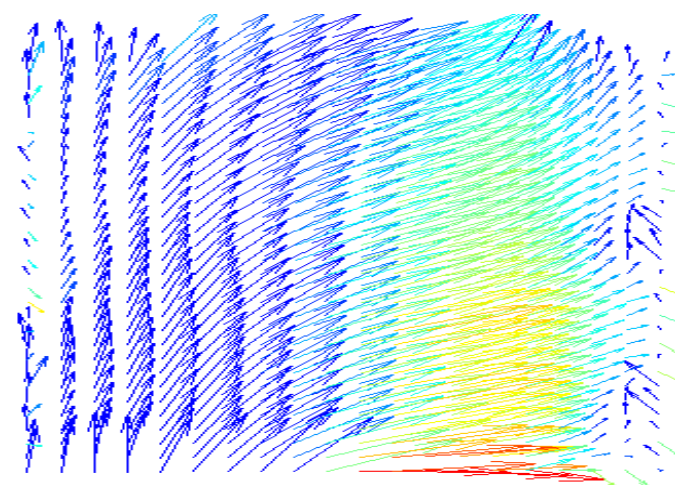

Figure 7: Velocity vector profiles with swirl flow along the test tube for $\mathrm{Re}=13000$

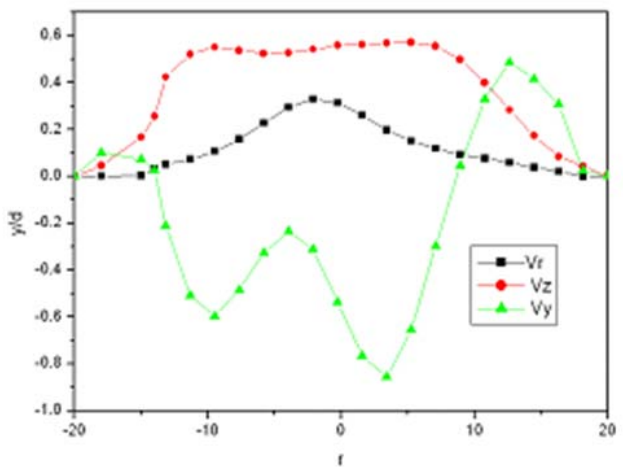

Figure 8: Velocity Profiles with swirl at $\mathrm{y} / \mathrm{d}=2$ for $\mathrm{Re}=13000$ 


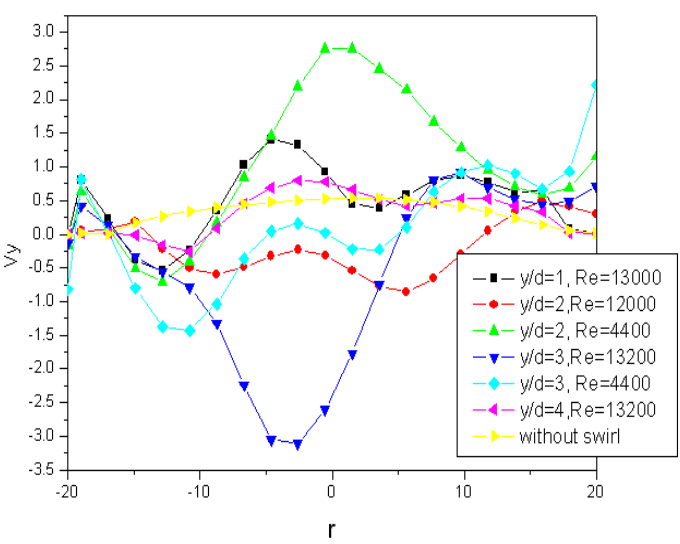

Figure 9: Comparisons of velocity profiles with and without swirl along the test tube

The vector with swirl for $\mathrm{Re}=13000$ along the test tube is demonstrated in Figure 7. As previously mentioned, the vectors had strong flow angles and showed negative values near the centerline of the test section for $\operatorname{Re}=13000$. However, the negative velocity vectors vanished by decaying along with the swirl intensity along the test section.

The local velocities with swirl for $\operatorname{Re}=13000$ in Figure 8. The Vy velocity is very similar to that in horizontal tube. However, the velocity profile is slightly smaller than in horizontal tube at identical Reynolds number. Moreover, the $\mathrm{Vz}$ velocity depicted is much lower than in horizontal tube. These phenomena are considered from the water gravity in the test tube.

The conditions with and without swirl for $\mathrm{Re}=$ 4400 to 13200 along the test tube were compared and are included in Figure 9.

The negative velocities showed strong values with increasing Reynolds number. But these velocities decayed along the test section with reducing swirl intensity.

Moreover, the flow moved up eccentrically along the vertical tube, indicating the decay of the tangential velocity.

\section{Conclusions}

1) The particles move toward the test tube wall with swirl and some reversal zones appear near the centerline of the test tube. These phenomena are very similar to those in a horizontal circular tube.

2) The local velocity ( Vy) indicates a negative value near the centerline of the test tube and positive values near the wall.

The negative velocity changes into positive along the test section with increasing swirl intensity or Re number.

3) The $\mathrm{Vz}$ velocity depicted is much lower than in horizontal tube. These phenomena are considered from the water gravity in the test tube.

\section{ACKNOWLEDGMENTS}

This present study was supported financially by the Ministry of Education, Science and Technology along with the Korean Institute of Science and Technology Information as a result of the RESEAT program.

\section{References}

[1] John B. Nuttall, “Axial flow in a vortex”, NATURE, vol. 172, no. 4378, pp. 582-583, 1953.

[2] A. M. Binnie and J. D. Teare, "Experiments on the flow of swirling water through a pressure nozzle and open trumpet”, Quart J. of Mech. And applied Math., vol. X, part. 3, pp. 78-89, 1957.

[3] A.M. Binne, "Experiments on the swirling flow of water in a vertical pipe and a bend", AIAA JOURNAL, pp. 452-465, 1962.

[4] R.J.K. Wood, T.E. Jones, N.J. Miles and J. Ganeshalingam, “Upstream swirl-induction for reduction of erosion damage from slurries in pipeline bends”, Wear 250, pp. 770-778, 2001.

[5] H. Mak and S. Balahani, "Near field characteristics of swirling flow past a sudden expansion”, Chemical Engineering Science vol. 
62, pp. 6726-6746, 2007.

[6] Shyy Woei Chang and Tsun Lirng Yang, "Forced convective flow and heat transfer of upward concurrent air-water slug flow in vertical plain and swirl tubes”, Experimental Thermal and Fluid Science, vol. 33, pp. 1087-1099, 2009.

[7] Doh, D.H, Hwang, T.G. and Saga, T., “3D-PTV measurements of the wake of a sphere”, Measurement Science and Technology, vol. 15, pp. 1059-1066, 2004.

[8] Soloff, S. M., Adrian, R. J., and Liu, Z. C., "Distortion compensation for generalized stereoscopic particle image velocimetry", Measurement Science of Technology, vol. 8, no. 12, pp. 1441-1453, 1997.

[9] Kimura, I., Takamori, T. and Inoue, T., "Image processing instrumentation of flowby using correlation technique”, Journal of Flow Visualization and Image Processing, vol. 6, no. 22, pp. 105-108, 1986.

\section{Author Profile}

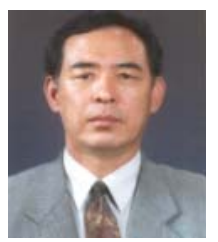

\section{Tae-Hyun Chang}

Tae-Hyun Chang, He was educated at Dong-A University(B. E., 1969, M. E. 1971) in Korea. He worked for Busan Thermal Power Plant as mechanical engineer. He obtained his Ph.D at Depart. mear of mechanical engineering of Univ. of Wales (U.K). He worked for Kyungnam Univ. for 30 years at the Division of mechanical and automation as a Professor. Now, he has worked for Korea Institute of Science and Technology Information as a senior research fellow. His major research field is convection heat transfer with and without swirl flow.

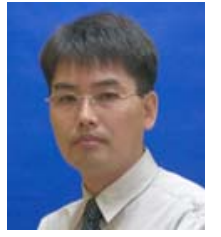

\section{Chang-Hoan Lee}

He obtained his B.S.C and M.S.C at Yonsei University and Ph.D at University of Seoul on 2008 and then he worked for North Carolina State University in USA as a research scholar. Now, he has worked for Korea Institute of Science and Technology Information as a Principal Researcher for 20 years. His interesting field is the information analysis of science and technology with papers and patents to find the international competitiveness of science and technology, a technology position and so on. 